ISAHP 1996, Vancouver, Canada, July 12-15, 1996

\title{
A METHOD TO REFINE PRIORITY OF OBJECTIVE CRITERIA IN AHP ${ }^{\text {1 }}$
}

\author{
Kazutomo Nishizawa \\ Department of Mathematical Engineering, College of Industrial Technology, \\ Nihon University, 1-2-1, Izumicho, Narashino, Chiba, 275, Japan \\ nisizawa@su.cit.nihon-u.ac.jp
}

\begin{abstract}
AHP (Analytic Hierarchy Process) is a useful tool for decision-makers, however, results by AHP do not coincide perfectly with the actual order of activities. The cause of differences depends on the comparison of objective criteria but not on comparison of activities. Because the former is very delicate and unstable and the latter is rather stable, it is not easy to decide the priority of objective criteria. In this paper, we propose a method to refine priority of objective criteria based on calculated weight from comparison matrices and known actual order of activities.
\end{abstract}

\section{Introduction}

In this paper, using AHP (Analytic Hierarchy Process) (Saaty,1977), we propose a method to refine the priority of objective criteria based on calculated weight from comparison matrices and actual order of activities.

AHP is a useful tool for the decision-maker and is applied to predict the order of activities. However, the result by AHP does not always coincide with the actual order. The cause of differences depends on the comparison of objective criteria and not on comparison of activities. Because the former is very delicate and unstable and the latter is rather stable, it is not easy to decide the priority of objective criteria. Further, a study with respect to priority of criteria in AHP has not been examined in detail.

This paper refines the priority of criteria based on calculated weight and actual order of activities. The following is the outline of our method. With respect to each activity we construct comparison matrices based on calculated weight and apply the eigenvalue method of AHP. Based on the result of the weights, we refine the priority of criteria.

In order to confirm the usefulness of our method, we apply our method to an example. First, based on actual order of activities, we define the priority of criteria. Next by the usual method, we calculate the weight of activities based on the refined priorities of criteria. We then compare the resulting weights of activities with the actual order of activities.

In section 2, we describe how to refine priority of objective criteria in detail and in section 3 we apply our method to an example. Finally, in section 4, we conclude our investigation.

\section{Refine Priority of Objective Criteria}

We consider typical complete three-level AHP hierarchy, shown in Fig. 2.1, consisting of $m$ objective criteria $c,(\mathrm{j}=1 \sim m)$, and $n$ activities $a,(\mathrm{j}=1 \sim n)$. By the ordinary procedure of AHP, we have the weight of activities, $x,(j=1 \sim n)$, and decide the order of activities.

\footnotetext{
${ }^{1}$ Acknowledgments: The author wishes to thank Dr. Iwaro Takahashi, Professor of Nihon University, for his useful advice on this investigation. The author is grateful to the referees for their valuable and helpful comments.
} 


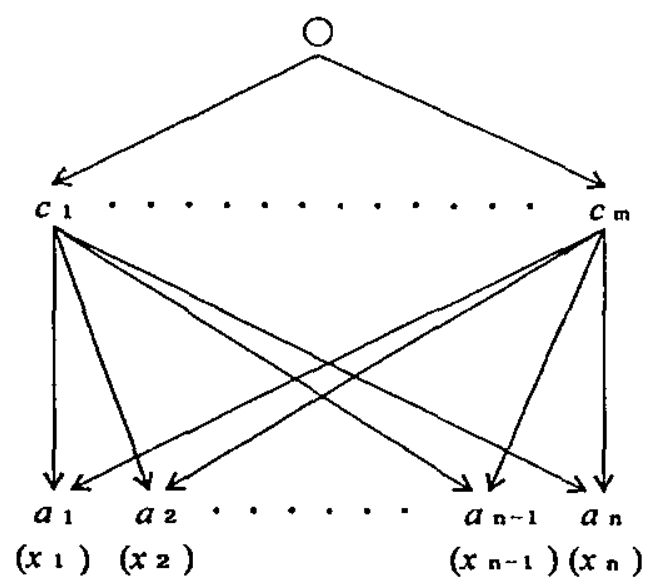

Fig. 2.1 Typical Complete Three-Level Hierarchy

Firstly, for each $\mathrm{i}=1 \sim m$ we construct comparison matrix $A_{1}$, with respect to criterion $\mathrm{c}_{1}$. The element $A,(\alpha, \beta)$ represents the result of comparison between $a_{\alpha}$ and $a_{s}(\alpha, \beta=1 \sim n)$. From matrix $A$ i we have weight $w$ is $(\mathrm{j}=1 \sim n)$ as $\mathrm{j}$-th element of the principal eigenvector of $A \mathrm{i}$, where

$$
\sum_{j=1}^{n} w i s=1 \text {. }
$$

Secondly, we construct comparison matrix $C$, whose element $C(\alpha, \beta)$ represents the result of comparison between $c_{\alpha}$ and $c_{s}(\alpha, \beta=1 \sim m)$. From matrix $C$ we have weight $v_{1}(\mathrm{i}=1 \sim m)$ as $\mathrm{i}$-th element of the principal eigenvector of $C$, where

$$
\sum_{i=1}^{m} v_{i}=1
$$

Then we have the weight $x ;(j=1 \sim n)$,

$$
x s=\sum_{i=1}^{m}\left(w_{i} x_{v i}\right), \text { where } \sum_{j=1}^{n} x_{1}=1,
$$

and we can decide the order of activities.

Generally the comparison matrices $A_{1}(\mathrm{i}=1 \sim m)$ are consistent but $C$ is often inconsistent and unstable. On the other hand, the actual weight of activities, $y,(j=1 \sim n)$, are often known, where

$$
\sum_{\mathrm{j}=1}^{n} y_{1}=1 \text {, }
$$

then we can refine priority of objective criteria $c$ । $(\mathrm{i}=1 \sim m)$. We illustrate this idea in Fig. 2.2.

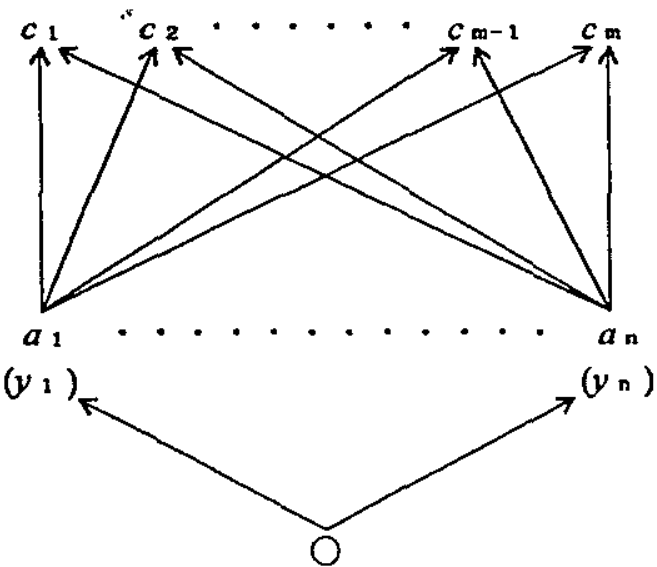

Fig. 2.2 Refine Priority of Criteria

For each $j=1 \sim n$, we can construct comparison matrix $A^{\prime}{ }_{j}$ with respect to activity $a_{j}$, using calculated weight $w$ is $(\mathrm{i}=1 \sim m)$. The $(\alpha, \beta)$ element of $A^{\prime}$, is defined as follows:

$$
A^{\prime},(\alpha, \beta)=w_{\alpha} / w_{B} \text { s }(\alpha, \beta=1 \sim m) \text {. }
$$

From matrix $A^{\prime}$, we have weight $w^{\prime}$, $(\mathrm{i}=\mathrm{l} \sim m)$ as $\mathrm{i}$-th element of the principal eigenvector of 


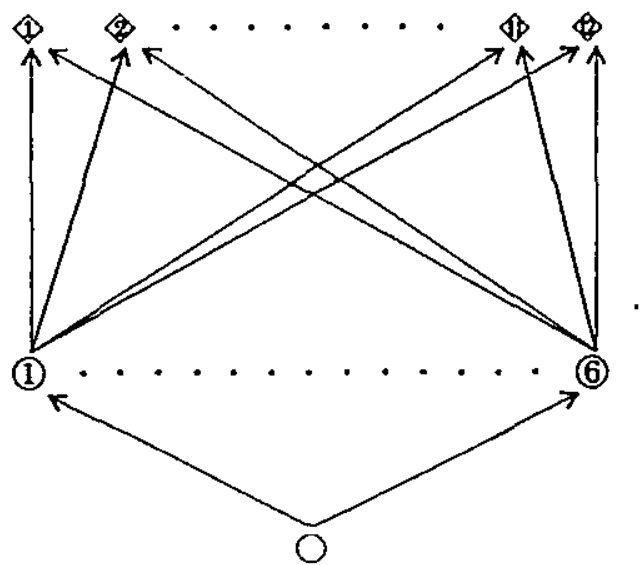

Fig. 3.2 Refine Priority of Criteria for Example

For criterion 1

$$
A_{1}=\text { (3) }\left[\begin{array}{llllll}
1 & \text { (2) } & \text { (3) } & \text { (4) } & \text { (5) } & \text { (6) } \\
\text { (1) } & \theta & \theta & \theta^{-1} & \theta & \theta \\
\theta^{-1} & 1 & \theta & \theta^{-1} & \theta^{-1} & \theta^{-1} \\
\theta^{-1} & \theta^{-1} & 1 & \theta^{-1} & \theta^{-1} & \theta^{-1} \\
\theta & \theta & \theta & 1 & \theta & \theta \\
\theta^{-1} & \theta & \theta & \theta^{-1} & 1 & 1 \\
\theta^{-1} & \theta & \theta & \theta^{-1} & 1 & 1
\end{array}\right]
$$

\begin{tabular}{|c|c|c|c|c|c|c|}
\hline & (1) & (2) & (3) & (4) & (5) & (6) \\
\hline (1) & 0.220 & 0.110 & 0.087 & 0.277 & 0.153 & 0.153 \\
\hline (b) & 0.218 & 0.275 & 0.173 & 0.138 & 0.109 & 0.087 \\
\hline$\beta$ & 0.138 & 0.218 & 0.173 & 0.275 & 0.109 & 0.087 \\
\hline$\Leftrightarrow$ & 0.275 & 0.109 & 0.173 & 0.218 & 0.138 & 0.087 \\
\hline (5) & 0.275 & 0.087 & 0.218 & 0.109 & 0.173 & $\therefore 0.138$ \\
\hline (6) & 0.138 & 0.218 & 0.173 & 0.275 & 0.109 & 0.087 \\
\hline$\phi$ & 0.275 & 0.087 & 0.173 & 0.218 & 0.138 & 0.109 \\
\hline (2) & 0.275 & 0.138 & 0.218 & 0.109 & 0.173 & -0.087 \\
\hline$\phi$ & 0.173 & 0.087 & 0.218 & $0.275^{-}$ & 0.138 & 0.109 \\
\hline (1) & 0.275 & 0.109 & 0.218 & 0.173 & 0.138 & 0.087 \\
\hline 1 & 0.218 & 0.087 & 0.275 & 0.173 & 0.138 & 0.109 \\
\hline$\Leftrightarrow$ & 0.218 & 0.138 & 0.173 & 0.275 & 0.087 & 0.109 \\
\hline
\end{tabular}

By similar procedure, for each criterion, we can construct binary comparison matrices $A_{1}(\mathrm{i}=1 \sim, 12)$ and calculate weight $w_{i j}(\mathrm{i}=1 \sim 12, \mathrm{j}=1 \sim 6)$, where $\theta=2$. The results are shown in Table 4 .

Table 4 Calculated Weight $w$ I $(i=1 \sim 12, j=1 \sim 6)$

Of course for each $i=1 \sim 12, \sum_{j=1}^{6} w i j=1$.

Next for team (1), based on Table 4 and equation (2.5), we have matrix $A^{\prime}{ }_{1}$ as shown (3:2).

For each $\mathrm{j}=1 \sim 6$, we can construct matrices $A^{\prime} j^{\prime}$ and have weight $w^{\prime \prime}$ is $(\mathrm{i}=1 \sim 12)$ as shown in Table 5.

Of course for each $j=1 \sim 6, \sum_{i=1}^{12} w^{\prime}{ }_{11}=1$.

On the other hand, normalizing with the sum of the winning average in Table 1 equal to 1 , we have an actual weight of teams $y,(j=1 \sim n)$. Of course, $x^{\prime}$, is equal to $y$ s. The result is shown in Table 6 . 


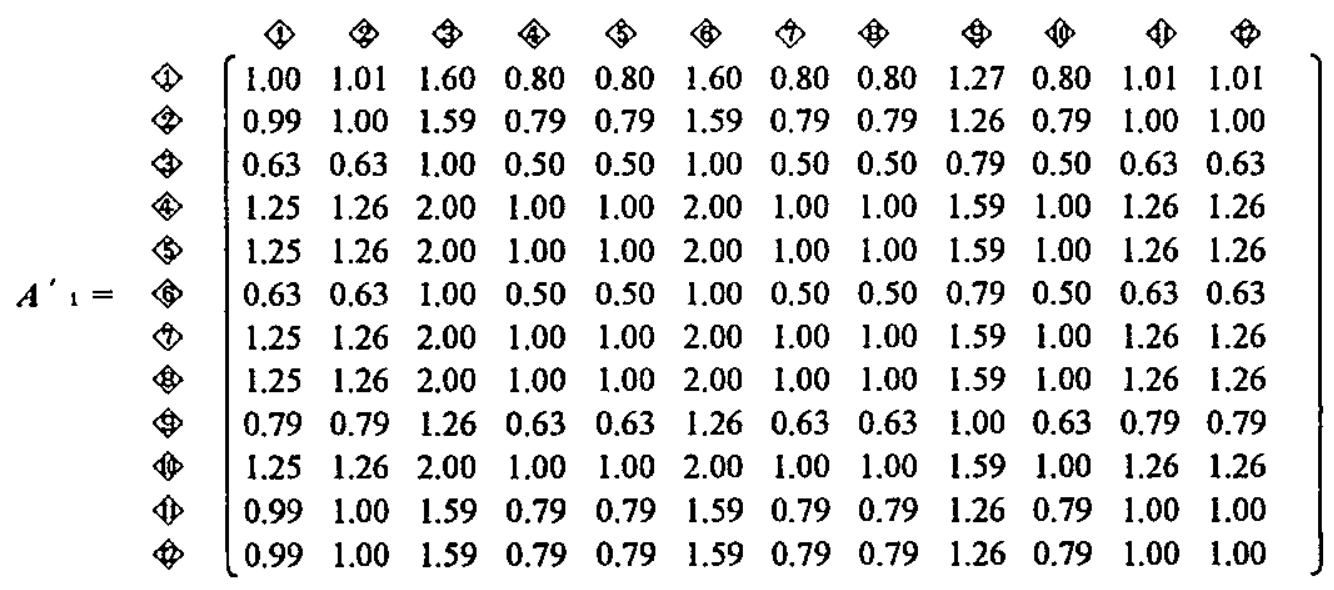

Table 5 Calculated Weight $w^{\prime}$ is $(i=1 \sim 12, j=1 \sim 6)$

\begin{tabular}{|c|c|c|c|c|c|c|}
\hline & (1) & (2) & (3) & (4) & (5) & (6) \\
\hline (1) & 0.081 & 0.066 & 0.038 & 0.110 & 0.095 & 0.124 \\
\hline (2) & 0.081 & 0.166 & 0.076 & 0.056 & 0.068 & 0.069 \\
\hline (9) & 0.051 & 0.131 & 0.076 & 0.109 & 0.068 & 0.069 \\
\hline$\Leftrightarrow$ & 0.102 & 0.066 & 0.076 & 0.087 & 0.086 & 0.069 \\
\hline (1) & 0.102 & 0.052 & 0.096 & 0.043 & 0.108 & 0.110 \\
\hline (6) & 0.051 & 0.131 & 0.076 & 0.109 & 0.068 & 0.069 \\
\hline (4) & 0.102 & 0.052 & 0.076 & 0.087 & 0.086 & 0.088 \\
\hline$\Leftrightarrow$ & 0.102 & 0.083 & 0.096 & 0.043 & 0.108 & 0.069 \\
\hline (9) & 0.064 & 0.052 & 0.096 & 0.109 & 0.086 & 0.088 \\
\hline (1) & 0.102 & 0.066 & 0.096 & 0.069 & 0.086 & 0.069 \\
\hline$\Delta$ & 0.081 & 0.052 & 0.122 & 0.069 & $0: 086$ & 0.088 \\
\hline (1) & 0.081 & 0.083 & 0.076 & 0.109 & 0.055 & 0.088 \\
\hline
\end{tabular}

Table 6 Calculated Weight $x^{\prime},(j=1 \sim 6)$

\begin{tabular}{rrrrrr}
\hline (1) & (2) & (3) & (4) & (5) & (6) \\
\hline 0.212 & 0.143 & $0.181^{\circ}$ & 0.180 & 0.155 & 0.129 \\
\hline & $\cdot$ & & & &
\end{tabular}

Of course $\sum_{j=1}^{6} x^{\prime},=1$

Finally, based on Table 5. Table 6, and equation (2.9), we have the weight $v^{\prime},(i=1 \sim 12$ ), shown in descending order in Table 7.

Table 7 Calculated Weight $v^{\prime}$ i $(i=1 \sim 12)$

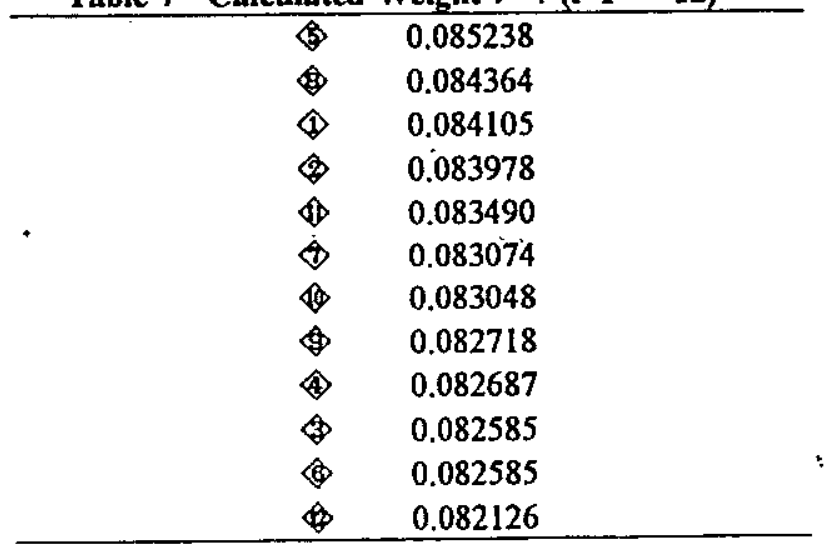

Thus we have priority of objective criteria, 1 , as shown in Table 7 . 
Now we can verify the results of our method. Based on the priority of criteria in Table 7, we are able to calculate the weight of teams by the AHP procedure and compare with the final standings in Table 1 .

Firstly, we calculate the weight of criteria. We can construct the binary comparison in matrix $C$, based on Table 7, which is shown in (3.3).

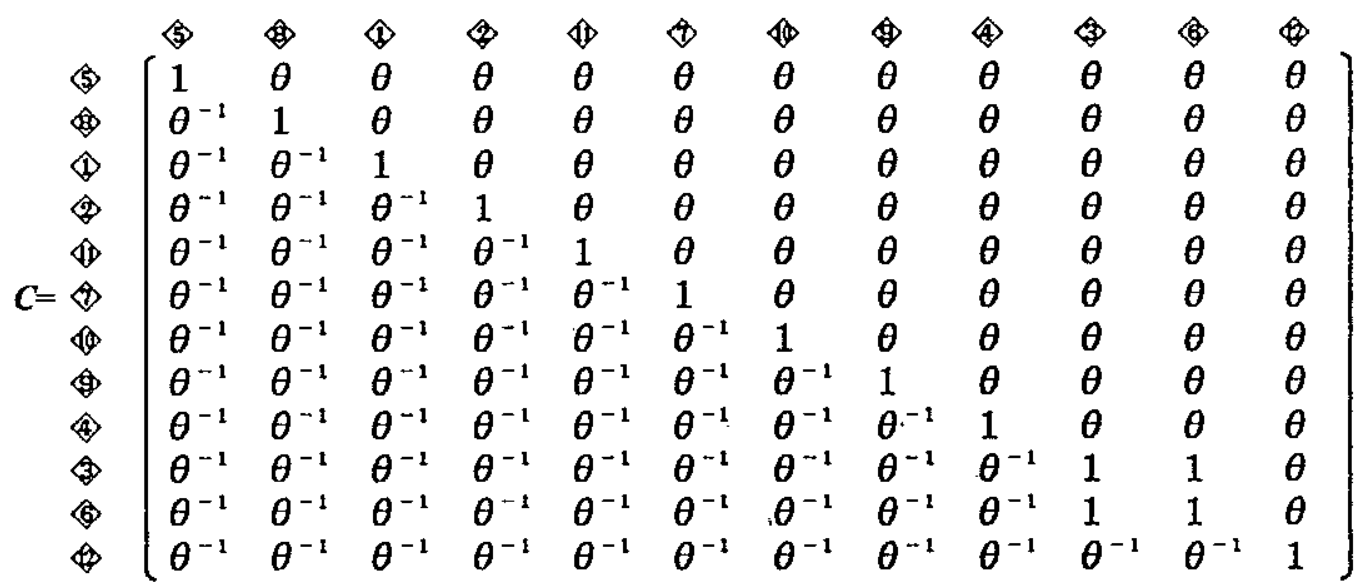

From the above matrix, where $\theta=2$, we have the weight $v_{1}(\mathrm{i}=1 \sim 12)$ shown in descending order in Table 8.

Table 8 Weight of Criteria

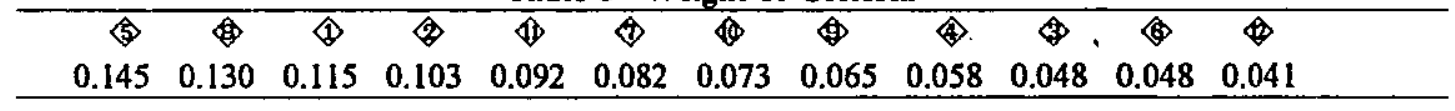

Then, using $w$ is $(\mathrm{i}=1 \sim 12, \mathrm{j}=1 \sim 6)$ in Table 4, $v_{1}(\mathrm{i}=\mathrm{I} \sim 12)$ in Table 8, and equation (2.3) we have the weight of teams. The results are shown in Table 9.

Tablè 9 Rèsult of Verification

\begin{tabular}{cc}
\hline final standings and weight & calculated ranking and weight \\
\hline (1) 0.212 & (1) 0.236 \\
(3) 0.181 & (3) 0.191 \\
(4) 0.180 & (4) 0.190 \\
(5) 0.155 & (5) 0.142 \\
(2) 0.143 & (2) 0.133 \\
(6) 0.129 & (6) 0.108 \\
\hline
\end{tabular}

As a result, in Table 9, the calculated ranking coincides with the final standings.

\section{Conclusion}

In this paper we proposed a method to refine the priority of objective criteria based on calculated weight and actual weight of activities. By applying our method to an example, we were able to illustrate and refine the priority of criteria. Further, by using AHP and using the refined priority of criteria, calculations were carried out along with the weight of activities. As a result, the calculated ranking of activities coincides with the actual ranking. Thus, we illustrate the effectiveness of the proposed method. However, we may have missed some important criteria. Therefore, there need to improve our method for future studies. 
Saaty, T. L. (1977) "A Scaling Method for Priorities in Hierarchical Structures", Joumal of Mathematical Psychology, 15, 234-281.

Takahashi, I. (1990) "AHP Applied to Binary and Temary Comparisons", Journal of Operations Research Society of Japan, 33, No. 3, 199-206.

Takahashi, I. and Fukuda, M. (1991) "Comparisons of AHP with other methods in binary paired comparisons", Proceedings of the Second Conference of the Association of Asian-Pacific Operational Research Societies within IFORS, 325-331.

Nishizawa, K. (1995) "A Consistency Improving Method in Binary AHP", Joumal of Operations Research Society of Japan, 38, No. 1, 21-33. 